\title{
Principles of Individual Quantitative Assessment of the Effect of Drugs in Diabetes According to the Akabane Test
}

\section{Muzhikov $\mathbf{V}^{1 *}$, Vershinina $\mathrm{E}^{2}$ and Muzhikov $\mathbf{R}^{3}$}

${ }^{1} \mathrm{CEO}$ of the Medical Department, Russia

${ }^{2}$ Laboratory of Information Technologies, Pavlov Institute of Physiology, Russia

${ }^{3}$ Software Engineering, Russia

*Corresponding author: Valery Muzhikov, M.D, Ph.D, CEO of the Medical Department,

\section{Research Article \\ Volume 5 Issue 1}

Received Date: February 15, 2021

Published Date: March 01, 2021

DOI: $10.23880 /$ ipcm-16000212 Gzhatskaya str. 5/3-139, St. Petersburg, Russia, Tel: +79627228981; Email: mujikov_v@mail.ru

\section{Abstract}

Background: Evaluation of drug action is a complex, expensive procedure that usually takes several years and is performed on a large group of subjects. However, as a result, this does not exclude the possibility that at the individual level, the positive effect of the drug will be minimal, and the side effects will be very large. A study on the example of the action of insulin in diabetes is devoted to the creation of a system of metrological assessment of the action of a drug at an individual level, including an assessment of its effectiveness, risks, optimal dose and time of administration.

Methods: In the clinic of endocrinology, Akabane tests were performed on 225 patients, including 120 women and 105 men with type 1 and type 2 diabetes (T1D and T2D) at different times after injecting various doses of insulin for short and longactions, including in the dynamics of observation.

Results: During the monitoring of patients with diabetes, it was found that under the influence of diabetes and various other diseases, asymmetry occurs in the indicators of certain acupuncture channels, and the optimal individually effective drug leads to the restoration of their symmetry. Based on this principle, it is proposed to evaluate the effect of a drug at an individual level through the coefficients of asymmetry and adhesion, which makes it possible to evaluate the effectiveness of a drug on new physical principles.

Conclusion: The use of the proposed system allows for a quantitative cumulative assessment of both positive and negative effects of a medicine on various organs and systems at an individual level. Such an assessment can be carried out after 2-3 doses, assess the overall effectiveness of the drug; select its optimal dose and time of administration. This principle also allows you to create a family of specialized miniature devices for these purposes.

Keywords: Effect of Drugs and their Doses; Glycaemic Control; Insulin; Optimal Timing of Medication; Symmetry and Asymmetry in the Body; Akabane Test

Abbreviations: AC: Acupuncture Channels; TCM: Traditional Chinese Medicine; LED: Light-Emitting Diode; TS: Temperature Sensitivity; IR: Infrared Radiation; SEM: Standard Error of the Mean; T1D: Diabetes Mellitus Type 1; T2D: Diabetes Mellitus Type 2; C: Coefficient.

\section{Introduction}

Currently, the individual selection of drugs and their dose is determined on the basis of group statistical studies on the diagnosis of the disease and the effect of influence 


\section{International Journal of Pharmacognosy \& Chinese Medicine}

on various key parameters of the body. Such a procedure requires the creation of a large amount of data and is highly stretched in time, often up to several years. However, this does not exclude the possibility that at the individual level this drug and its standard dose may not give the desired effect, or that its use will cause undesirable complications that exceed its benefits.

Therefore, there is an urgent need to create a system of certain metric indicators with a quick response after 1-2 uses of the drug, which would demonstrate its effectiveness at the individual level. However, such a decision requires a revision of the basic principles for assessing the effect of drugs that exist now. In this regard, we were attracted by the principle of assessing harmony in living nature, based on the rules of symmetry and dissymmetry [1-4].

In nature, all inanimate tends to symmetry and repeating structures, for example, at the level of Mandelbrot fractals Living matter is also symmetrical, for example, at the level of right/ left, but this axial symmetry is violated during illness. For example, normally the leaf of a plant is symmetrical about the midline, but in the case of a plant disease, an asymmetric deformation, spots and other violations of the structure of symmetry appear in the leaf. Thus, this fundamental universal criterion can become an arbiter in assessing the presence of a disease. The more pronounced the disease, the higher the violation of symmetry in certain fractals of living matter.

However, how to measure the violation of symmetry at the level of a person and especially at the level of its individual organs and systems? The answer to this question can be obtained from the TCM, where the bioenergetics of the body has been evaluated for more than one hundred years at the level of acupuncture channels (AC). for the detection of various diseases. In particular, the Akabane test and its analogues are used to assess it $[5,6]$.

To quantitatively evaluate a channel's activity level in TCM, the so-called "sacrificial stick" test was used in ancient times in China. This test involved a burning sandal stick that was brought in proximity to points at the tips of every finger and toe until the first sensation of pain. At each point, the pulse rate before pain was first felt, was measured. If the number of pulse beats was lower than the average one for all the channels, it proved the hyperactivity of an acupuncture channel and its corresponding organ. Conversely, when the rate was higher, it represented the inactivity of a channel and its corresponding organ. This test was described by the Japanese doctor Koben Akabane in 1956. Since then, the test has carried his name. To perform this test, we use measures the pain thresholds in temperature sensitivity (TS) when heat is applied to the "entrance-exit" points of each channel
(LU11, LII1, PC9, TE1, HT9, SI1, SP1, LR1, ST45, GB44, KI1, and BL67) by applying an impulse light-emitting diode (LED) non-coherent infrared radiation (IR)-light onto the skin ( $\mathrm{f}=1$ $\mathrm{Hz}, \lambda=920 \mathrm{nM})$, recording the total energy expenditure in joules. Each impulse radiates 0.07 joules of thermal energy. These measurements were carried out using a certified device "Merid", invented by the author of the paper. TS are our bodies' basic reactive system; it is as significant as important indicators such as body temperature, as it gives a very clear representation of functional and psycho-physiological profiles. Their topography is shown in Figure 1.

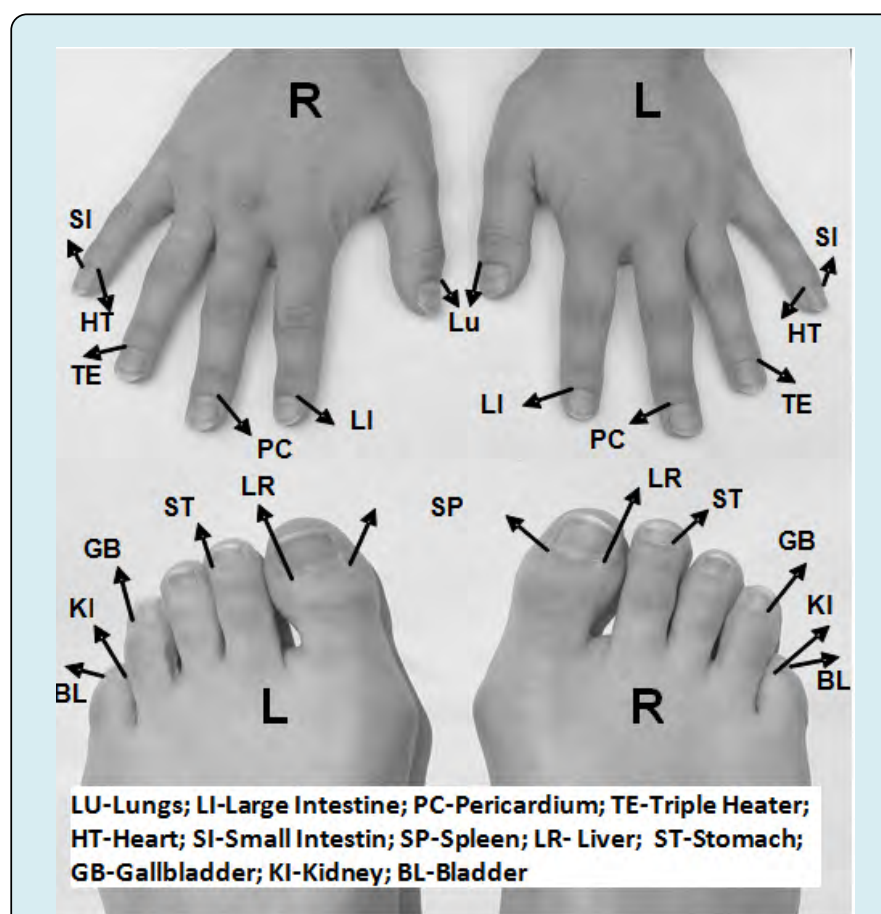

Figure 1: Topography of acupuncture points, used for evaluation of the AC in Akabane test.

According to classical Oriental literature as well as according to our study results $[3,4]$, the following channels have different regulatory functions:

LU- Lungs channel, connected with the function of lungs and tissue breathing.

Li-Large intestine channel, connected with the large intestine function and its microbe flora, it participates in the regulation of arterial pressure, biochemical blood indices.

PC-Pericardium channel, connected with the cardiac muscle trophicity and its structure. In addition, it is connected to muscular activity, arterial blood pressure levels and emotions.

TE-Triple heater channel, connected to the central and peripheral hemodynamic, and with body energy consumption. In addition, it regulates the hormones in the body, thyroid and hypophysis. 


\section{International Journal of Pharmacognosy \& Chinese Medicine}

HT-Heart channel, regulates the cardiac rhythm, body's physical strength

Si-Small intestine channel, connected to the electrolytic balance and food digestion.

SP-Spleen-pancreas channel is connected to the pancreas and immunity.

LR-Liver channel is connected to liver function and central nervous system, stress levels

St-Stomach channel is connected to the digestion function.

GB-Channel of the gallbladder is connected to the digestion function and peripheral nervous system.

$\mathbf{K i}$-Kidney channel is connected to the kidney function and the adrenal glands.

BL-Urinary bladder channel is connected to the urogenital system, its functions and its related hormones, it reflects the state of vertebrae column as well.

Each channel consists of the left (l) and the right (r) branch between which normal symmetrical activity balance is maintained.

Since each AC has a right and left branch and all points on the body are symmetrical in localization, the Akabane test is an ideal tool for controlling the level of symmetry, quantifying it at the TS level at certain points.

For example, in diabetes, there is a violation of symmetry at the level of certain $\mathrm{AC}$, and the degree of violation of symmetry is generally proportional to the degree of violation of carbohydrate metabolism [7-10]. On the other hand, a properly selected medicine provides healing and thus restores symmetry in the body [11-13].

However, the problem is that not all organs in our body are symmetrical. The liver, stomach, pancreas, spleen are represented in 1 number and are not symmetrically located relative to the midline of the body. How to apply this concept in this case? Traditionally, in TCM it was believed that, for example, the common spleen-pancreas channel has common symmetrical diagnostic points RP1, moreover, the right point reflects the state of the pancreas, and the left one reflects the spleen. And for example, the canal of the liver and stomach, when measured from the right and left points, reflects the functional activity of different anatomical parts of these organs.

However, earlier Muzhikov V, et al. [7,12] showed a general model of relationships between the sensitivity threshold of a number of ACs with the regulation of glycemic levels in patients with type 1 diabetes mellitus, which was in good agreement with the results of subsequent studies $[7,9]$. According to this model, on the pancreatic canal, the test indicators at the diagnostic point SP1 on the right and left separately reflect the total activity of B or A-cells of the pancreas.

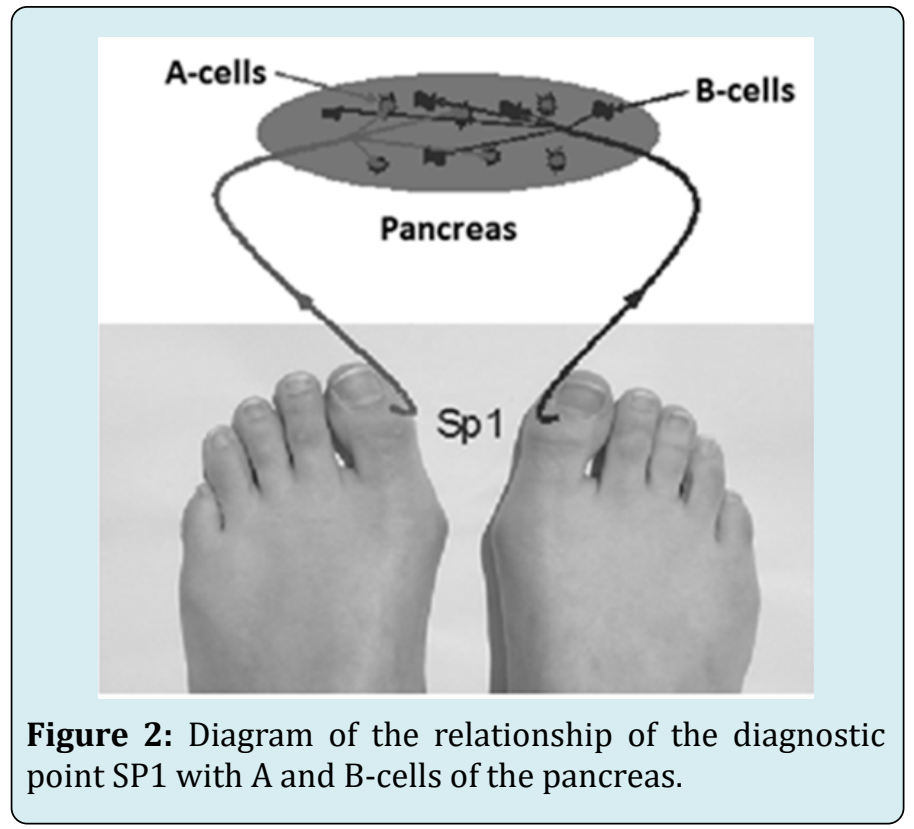

So the right point SPr reflects the activity of B-cells of the pancreas, which produce insulin and lower blood sugar levels. Conversely, the left point (SPl) reflects the activity of the counterinsular hormone, which increases glucose synthesis. Thus, due to the interaction of these two subsystems opposite in action, the blood sugar level is regulated within the normative corridor.

In type 1 diabetes with partially preserved function of B - cells $[7,9]$, there is a pronounced asymmetry of indicators with a predominance of $\mathrm{TS}$, especially the right branch of the SP channels, in proportion to the level of glycemia. If the activity of B cells is absent, then there is no asymmetry along the SP channel, including in the dynamics of observation with different glycemic parameters. However, in the presence of compensatory mechanisms, asymmetry appears in other AC.

This rule applies to both paired and non-paired organs and body systems. Everywhere there are 2 opposite regulatory subsystems: one increases its activity at the level of its specific parameter, and the other decreases it, and both are associated with one diagnostic point in which the overall regulatory balance is reflected proportion at the level of the right/left.

\section{Materials and Methods}

Akabane tests were performed on 225 patients, including 120 women and 105 men with type 1 and type 2 diabetes (T1D and T2D) at different times after injecting various doses of insulin for short and long- actions, including in the 


\section{International Journal of Pharmacognosy \& Chinese Medicine}

dynamics of observation.

The diagnosis of diabetes was confirmed on the basis of standard examination methods in a specialized endocrinology hospital. The average age of the patients was $48.71 \pm 14.2$ years. Patients with polyneuropathy were excluded from the study. For each patient, the effect of a short and long-acting insulin dose on the $\mathrm{AC}$ was analyzed separately, as well as the total insulin dose per day, the time after each dose, and the time after meals before the Akabane test. In these patients, the association of AC with glucose from capillary and venous blood was also analyzed.

All data are presented as mean \pm standard error of the mean (SEM). The profiles of TS were compared using a t- test for two independent samples and the post hoc tests and the Mann-Whitney U test. The analysis of the relations between AC was carried out using correlation (Pearson \& Spearmen coefficients). To evaluate the dependence channels on glucose multiple regression analysis was used in men and in women. Statistical decisions were made at a significance level of $5 \%(\mathrm{p} \leq 0.05)$. Data analysis was provided using the software package SPSS Inc. V 15 (SPSS Inc., Chicago, IL, USA).

\section{Results}

To confirm these theses indicated above, let us dwell on the results of observations on patients with diabetes, for whom the most effective drug is insulin in various modifications. However, they are not always suitable for specific patients.

The degree of asymmetry can be assessed through the ratio of the indicators of the right and left branches of their $\mathrm{AC}$ with the calculation of the asymmetry coefficient $\mathrm{K}$ (K=TS right/TS left of the AC branch). The optimal value for the norm is $\mathrm{K}=1.0$ or close to it. Therefore, this ratio of relationships is the most effective criterion that reflects the presence of regulatory failures at the level of various organs and systems [6-16]. Most clearly, these changes in T1D can be assessed by the profile of the K-asymmetry at the level of various AC [9].

So, Figure 3 shows the profile of such ratios in type 1 diabetes versus normal. It should be noted that normally there is also an asymmetry of $15-20 \%$ due to the predominance of the right branch over the left. This is due to the fact that the right branch of the AC (it is Yin according to the TCM definitions) reflects mainly anabolic processes and energy accumulation in the organ or physiological system connected to the AC, and the left branch (Yang according to TCM) reflects catabolic processes and energy expenditures, which are usually below the level of energy accumulation with normal nutrition $[11,12,16]$.
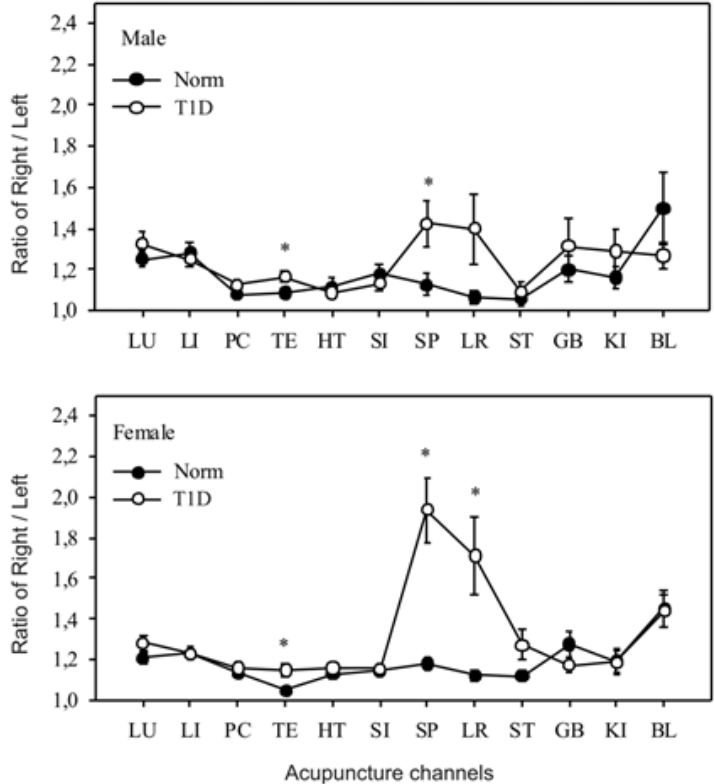

*Significant differences, t-test

Figure 3: Ratios of Right/Left STs of channels when comparing healthy subjects and those with T1D.

Both types of diabetes are generally characterized by a metabolic imbalance associated with regulatory failures in a number of systems and organs. Thus, in men with T1D, a surge in asymmetry was noted, especially at the level of the SP, LR, GB, KI channels. In women, the level of asymmetry is especially high along the SP, LR channels, up to $\mathrm{K}=2.0$.
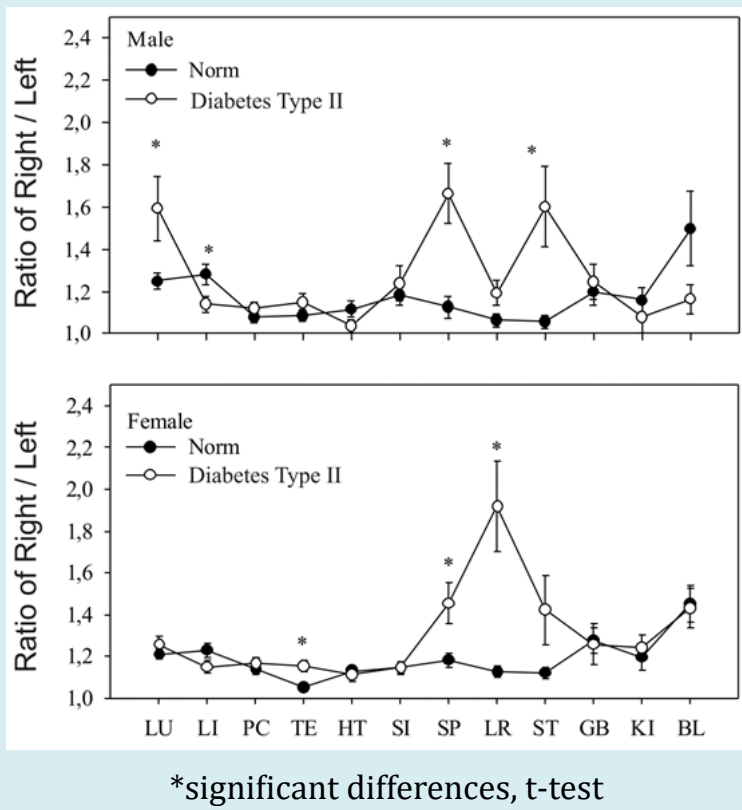

Figure 4: Ratios of Right/Left TS of channels when comparing healthy subjects and those with T2D. 


\section{International Journal of Pharmacognosy \& Chinese Medicine}

In T2D Figure 4, significant asymmetry compared with the norm in men was noted along the LU, LI, SP, ST channels, and in women, the asymmetry was especially pronounced along the LR, SP, ST channels [8,10]. At the same time, the level of deviation (spread) of these indicators often changes in proportion to the level of glycemia, which allows its indirect assessment by a calculated non-invasive method, which is very convenient, especially in the course of individual monitoring.

On the other hand, if the level of deviation changes in proportion to the change in the level of glycemia, then this AC is the most significant marker for monitoring and controlling the action of drugs. Thus, according to the test profile, in contrast to invasive glucose measurements, it is possible to simply and conveniently assess the degree of metabolic disorders at the level of individual organs and systems, for example, before and after meals, physical exertion and, which is especially important, after taking sugar-reducing drugs.

Table 1 shows the results at the level of the most reliable relationships in terms of the M-W and Post hoc test values in comparison of the indicators of the asymmetry coefficient of TS in patients with D1-2T with the norm in men and women [7].

So in type 1 diabetes, these are the links between the glycemic level and the TE, SP, LR channels. In type 2 diabetes, a similar, significant asymmetry is observed in AC-LI, SP, LR, ST, BL, which also changes with the level of glycemia. Overall, these results further clarify the most significant associations with AC in diabetes.

\begin{tabular}{|c|c|c|c|c|c|c|c|c|}
\hline \multirow{3}{*}{ AC } & \multicolumn{4}{|c|}{ T1D } & \multicolumn{4}{|c|}{ T2D } \\
\hline & \multicolumn{2}{|c|}{ Male } & \multicolumn{2}{|c|}{ Female } & \multicolumn{2}{|c|}{ Male } & \multicolumn{2}{|c|}{ Female } \\
\hline & Post hoc & M-W & Post hoc & M-W & Post hoc & M-W & Post hoc & M-W \\
\hline LUr/LUl & & & & & $*, 029$ & & & \\
\hline LIr/LIl & & & & & $*, 022$ & $* *, 009$ & ,068+ & ,079+ \\
\hline $\mathrm{PCr} / \mathrm{PCl}$ & & & & & & & & \\
\hline TEr/TEl & $*, 026$ & ,095+ & $* *, 004$ & ,067 & & & $* *, 003$ & $* *, 010$ \\
\hline $\mathrm{HTr} / \mathrm{HTl}$ & & & & & & $*, 045$ & & \\
\hline SIr/SIl & & & & & & & & \\
\hline $\mathrm{SPr} / \mathrm{SPl}$ & $*, 017$ & ,086+ & $* * *, 000$ & ,056+ & $* * *, 000$ & $* *, 010$ & $* *, 009$ & ,056+ \\
\hline $\mathrm{LRr} / \mathrm{LRl}$ &, $056+$ & & $* *, 002$ & &, $053+$ & & $* * *, 000$ & \\
\hline $\mathrm{STr} / \mathrm{STl}$ & & &, $057+$ & & $* *, 005$ & &, $074+$ & $*, 026$ \\
\hline $\mathrm{GBr} / \mathrm{GBl}$ & & $*, 047$ & & & & & & $* *, 005$ \\
\hline KIr/KIl & & & & & & $*, 018$ & & \\
\hline BLr/BLl & & & & & ,084+ & $* *, 004$ & &, $082+$ \\
\hline
\end{tabular}

Note: * - p<0,05, ** - p<0,01, *** - p<0,001, + - tendency.

Table 1: Paired comparisons using the post hoc tests and Mann-Whitney test in the comparison of TSs of healthy subjects and those with T1D and T2D in relation to Right/Left.

Since insulin and other similar drugs reduce the level of glycemia and normalize the condition, the rules of symmetry/ desymmentry apply to them as well. Therefore, on the basis of the method of restoring symmetry in certain AC under the action of a drug, it is possible to form the very principle of choosing a hypoglycemic drug and its adequate dose at the individual level.
So it was found that the increase and decrease in the level of glycemia in diabetes is reflected in the change in the parameters of TS of the same AC, but they either have different signs in the correlation and regression dependences, or the sides of the relationship $[17,18]$. Table 2 shows the results of such comparisons in groups of patients with type 1 and type 2 diabetes, where this relationship is usually observed. 


\section{International Journal of Pharmacognosy \& Chinese Medicine}

\begin{tabular}{|c|c|c|c|c|c|c|c|c|}
\hline \multirow{3}{*}{$\mathrm{AC}$} & \multicolumn{4}{|c|}{ T1D } & \multicolumn{4}{|c|}{ T2D } \\
\hline & \multicolumn{2}{|c|}{ Male } & \multicolumn{2}{|c|}{ Female } & \multicolumn{2}{|c|}{ Male } & \multicolumn{2}{|c|}{ Female } \\
\hline & Glucose & Insulin & Glucose & Insulin & Glucose & Insulin & Glucose & Insulin \\
\hline $\mathrm{LU}$ & -LUl** & $+\mathrm{LUr}$ & $+\mathrm{LUr} * *$ & - & -LUl** & $+\mathrm{LUl}$ & $+\mathrm{LUl}$ & -LUl \\
\hline LI & $+\operatorname{LIr}^{* *}$ & -LIr & $+\operatorname{LIr}^{* *}$ & -LIr & $+\mathrm{LIr}^{* *}$ & -LIr & - & + LIl \\
\hline $\mathrm{PC}$ & - & $+\mathrm{PCr}$ & $-\mathrm{PCl}$ & $+\mathrm{PCr}$ & - & - & - & $-\mathrm{PCl}$ \\
\hline $\mathrm{TE}$ & - & $+\mathrm{TEl}$ & $+\mathrm{TEr}^{* *}$ & $+\mathrm{TEl}$ & - & -TEl & -TEr* & -TEl \\
\hline $\mathrm{HT}$ & $+\mathrm{HTl}^{* *}$ & $-\mathrm{HTl}$ & - & $+\mathrm{HTl}$ & $-\mathrm{HTr}^{* *}$ & $+\mathrm{HTl}$ & $-\mathrm{HTl}^{* *}$ & $+\mathrm{HTl}$ \\
\hline \multirow{2}{*}{ SI } & $+\mathrm{SIr}^{*}$ & \multirow{2}{*}{+ SIl } & \multirow{2}{*}{ - } & + SIr & + SIr* & \multirow{2}{*}{+ SIr } & \multirow{2}{*}{ - } & \multirow{2}{*}{+ SIl } \\
\hline & $-\mathrm{SIl}^{*}$ & & & -SIl & $-\mathrm{SIl}^{*}$ & & & \\
\hline SP & $+\mathrm{SPr}^{* *}$ & $-\mathrm{SPr}$ & $+\mathrm{SPr} * *$ & -SPl & $+\mathrm{SPr}^{* *}$ & $-\mathrm{SPr}$ & $+\mathrm{SPr}{ }^{* *}$ & -SPl \\
\hline LR & -LRr** & $+\mathrm{LRr}$ & $-\mathrm{LRr}^{* *}$ & $+\mathrm{LRr}$ & $-\mathrm{LRl}^{* *}$ & $-\mathrm{LRr}$ & $+\mathrm{LRI}^{* *}$ & $+\mathrm{LRr}$ \\
\hline ST & $+\mathrm{STl} *$ & -STl & - & $+\mathrm{STr}$ & $+\mathrm{STr} *$ & $-\mathrm{STr}$ & $-\mathrm{STl}{ }^{* *}$ & $+\mathrm{STl}$ \\
\hline \multirow{2}{*}{ GB } & -GBr* & \multirow{2}{*}{$-\mathrm{GBl}$} & \multirow{2}{*}{$-\mathrm{GBr}^{*}$} & \multirow{2}{*}{$+\mathrm{GBr}$} & $+\mathrm{GBr}^{* *}$ & \multirow{2}{*}{$+\mathrm{GBl}$} & $+\mathrm{GBl}^{*}$ & \multirow{2}{*}{$-G B r$} \\
\hline & $+\mathrm{GBI}^{*}$ & & & & $-\mathrm{GBI}^{* *}$ & & $-\mathrm{GBI}^{* *}$ & \\
\hline KI & - & + KIl & $-\mathrm{KIr}^{* *}$ & $+\mathrm{KIr}$ & -KIr* & -KIl & -KIr* & $-\mathrm{KIl}$ \\
\hline BL & $+B L r^{*}$ & -BLl & $+\mathrm{BLl}^{* *}$ & $+\mathrm{BLl}$ & $+B L r^{*}$ & - & - & $+\mathrm{BLl}$ \\
\hline
\end{tabular}

*Sugar-capillary blood from a finger; ** Glucose from the vein

Table 2: The main relationship of the $\mathrm{AC}$ with blood sugar levels and insulin doses in diabetes mellitus.

Thus, 2 opposite vectors of influence on the same AC destroy this asymmetry or balance it, increasing the indicators TS of the opposite side. In this case, it was noticed that even high, but symmetric values of indicators on both branches of the $\mathrm{AC}$, arising immediately after the use of the drug, are then accompanied by their proportional mutual decrease already after 10-15 minutes to the standard values. At the same time, the biochemical or physiological indicator of the organism itself, which is associated with the activity of the given $\mathrm{AC}$, is normalized.

In general, based on the regularities obtained, the effect of the drug on the AC can be described by the following rules:

- If $\mathrm{K}$ is initially more (when $\mathrm{TSr}>\mathrm{TSl}$ ) or initially less than 1.0 (when $\mathrm{TSr}<\mathrm{TSl}$ ) and does not change in the observation dynamics, then it is concluded that the drug is not effective for a given organ or physiological system,

- If $\mathrm{K}$ decreases or increases in the case of asymmetry variant 2, but does not reach the value of 1.0, then its individual dose is not sufficient,

- If Kafter the use of the drug is less than 1.0, with 1 variant of asymmetry and more than 1.0 with the second variant of asymmetry, then conclude that its dose is excessive, and can be subsequently reduced taking into account this index until $\mathrm{K}$ is equal to 1.0 ,

- If $\mathrm{K}$ after the administration of the drug was initially close to the level of 1.0, and then during its maximum period of action began to increase in the first variant of asymmetry, or vice versa to fall, in the second variant of asymmetry, then at a value of 1.2-1.4 or 0.8-0.6 additional administration drug is necessary to maintain the normal corridor along this channel.

However, in a number of cases, non-compliance is noted, and as a rule, most drugs do not completely cover all asymmetries along the channels caused by a specific disease or their mixture of major and concomitant diseases. Also, as a rule, drugs also have side effects on certain organs, which should be taken into account when choosing them individually. For example, in men with T1D, the effect of insulin according to Table 2 is associated with the growth of the right branch of the PCr channel and the left branch of the TEl channel, which are not affected by glycemia. This effect causes its asymmetry, which can in turn negatively affect muscle activity through the PC channel, the activity of the thyroid gland and central hemodynamics through the TE channel, since any violation of symmetry is not desirable for the body. In women with type 1 diabetes, such an unbalanced effect of insulin takes place in the AC-of the small intestine along both of its branches - on the right and left. In women with diabetes mellitus 2, such an additional influence is, for example, through the channels BLl, SIl, Lil, which can also cause an undesirable bias, and various side effects that correspond to the saying "one we treat, the other we cripple". 


\section{International Journal of Pharmacognosy \& Chinese Medicine}

Of course, in each specific case, the individual characteristics of the organism must be taken into account. For this, it is usually sufficient to assess the profile of the activity of the AC based on the results of several background observations before taking the drug. So in type 2 diabetes, for example, in men according to Figure 4, asymmetry occurs, mainly along the right branches of the pancreatic canal (SP), lungs (LU) and stomach (ST), when the right branch predominates over the left, which leads to a sharp asymmetry of their proportions of the average ratio of the TS right/left to a value of 1.6-1.7. Taking into account, for example, this graph, an ideal drug should selectively act only along the channels of the pancreas (SP), lungs (LU) and stomach (ST), but in two ways:

- Also only on the right side, but with the sign (-). As a result, opposite vectors of influence of glycemia and insulin lead to a decrease in asymmetry on the right.

- Due to the growth of indicators of the opposite side, which was already discussed earlier.

Thus, a properly selected medicine should influence and adhere selectively only to those AC, the asymmetry of which is a consequence of the disease and balances its manifestation as a "key to the lock", restoring harmony in the body. To assess this relationship, we began to use the "adhesion coefficient - C", as the ratio of those ACs that are affected by the disease to the amount of ACs that are affected by a particular drug. Ideally, $\mathrm{C}=1.0$. If the adhesion is not complete, when the drug acts only on a part of the $\mathrm{AC}$, then to equalize all the asymmetries, additional drug is needed, which will provide complete adhesion in the aggregate?

Since the effect of a drug at the individual level is initially difficult to predict, we widely use regression analysis to solve this problem [7-13]. For this, the background period is initially estimated before its intake or, for example, during its use, but with a maximum interval after its intake, when its concentration is minimal. Conversely, the effect of insulin should be assessed at its peak. Based on the results of several measurements according to the Akabane test, a step-by-step regression is built, for example, the dependence of the TS channels on the level of glycemia based on its measurements with an individual glucometer. The effect of insulin dose on AC is assessed in a similar way. If the dose is stable, then the time after injection is used instead, since the drug concentration usually decreases over time. In both models, the relationship of these indicators with the values TS of the $\mathrm{AC}$ is assessed, for example, at a confidence level of $\mathrm{p}<0.05$. The adhesion coefficient $\mathrm{C}$ is then calculated.

Thus, the adhesion coefficient (C) can be used to assess at the individual level how effective a drug is as a whole. So, if we take the standard for medicine criterion of reliability in the regression model ( $p<0.05)$, then an increase in blood sugar leads to an increase in asymmetry, for example, in 6 channels, and insulin acts only on 4 of them. We calculate $\mathrm{C}=6 / 4=1.5$. This means that the adhesion is not complete, and additional medicine is needed to completely level the asymmetry. If $C=6 / 6=1$, then such treatment eliminates all negative manifestations of hyperglycemia. If insulin acts on 8 channels, then $C=6 / 8=0.75$. Therefore, there are side effects from the drug, which is manifested by the formation of a new asymmetry that is not favorable for the body. It means that we need to look for a new medicine to achieve $\mathrm{C}=1.0$ or closer to it. Another solution is the selection of an additional drug that reduces the asymmetry caused by the previous treatment.

Based on these rules, the following can be estimated:

- Individual effectiveness of the drug as a whole, as well as at the level of certain organs and systems of the body exposed to hyperglycemia, according to the coefficient of adhesion (C),

- Its optimal dose.

- The optimal multiplicity and time of its reception were estimated in order to maintain the asymmetry index within 1.0. The longest possible time during continuous monitoring. So in Fig. 4 schematically shows various options for assessing the optimal dose of insulin and the time of its administration in patients with type 1 diabetes with partially preserved function of pancreatic B-cells. In this case, the most effective insulin action can be monitored on the AC of the pancreas (SP).

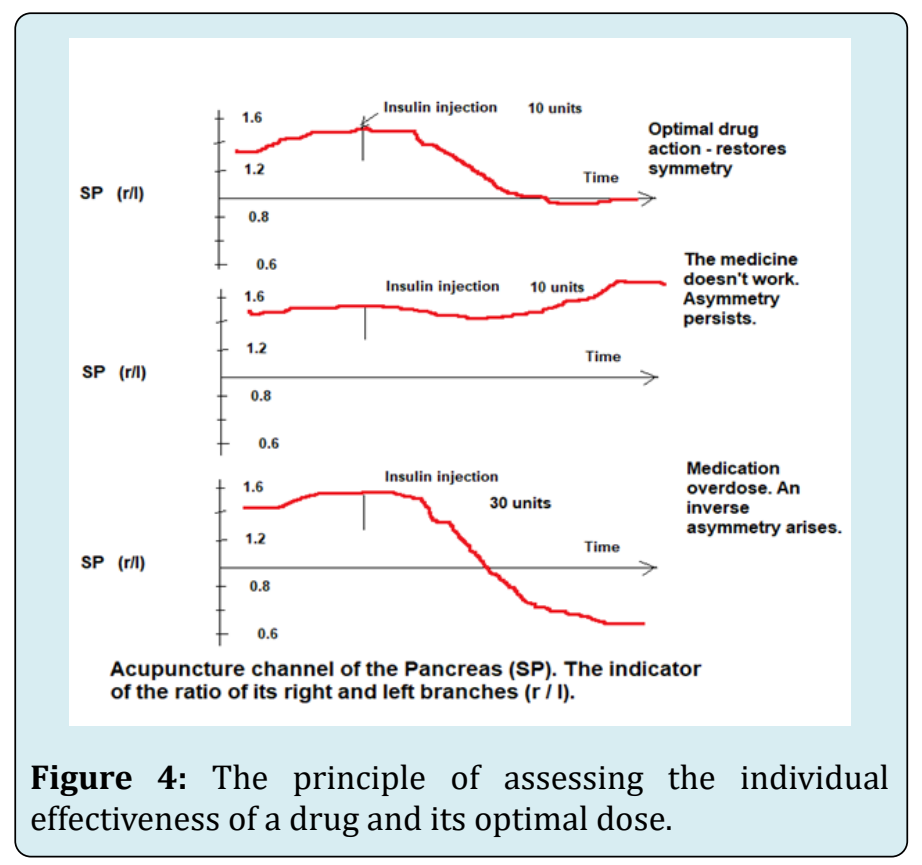

The first graph shows the optimal situation when after the introduction of 10 units. Insulin against the background 


\section{International Journal of Pharmacognosy \& Chinese Medicine}

of high asymmetry in the SR channel, characteristic of type 1 diabetes, the asymmetry coefficient $(\mathrm{K})$ is leveled to a level close to 1.0. The second graph shows a situation when the same dose of insulin does not affect a given AC. Therefore, such a drug is not effective. The lower graph shows the situation after the administration of 30 units of insulin. The result is an inverse asymmetry with $\mathrm{K}=0.7-0.6$, which characterizes overdose and hypoglycemia as a consequence.

To select the optimal time for drug administration, the situation is monitored, for example, according to 1 schedule. If the asymmetry increases above the individually comfortable corridor of the norm, then a new maintenance dose of the drug is needed so that the asymmetry value approaches 1.0.

Figure 5 shows the $\mathrm{r} / \mathrm{l}$ ratio profile at T2D diabetes in 3 ranges of glycemic levels (less than $7 \mathrm{mmol} / \mathrm{L}$, from $7-12 \mathrm{mmol} / \mathrm{L}$, and greater than $12 \mathrm{mmol} / \mathrm{L}$ ), compared with healthy subjects.

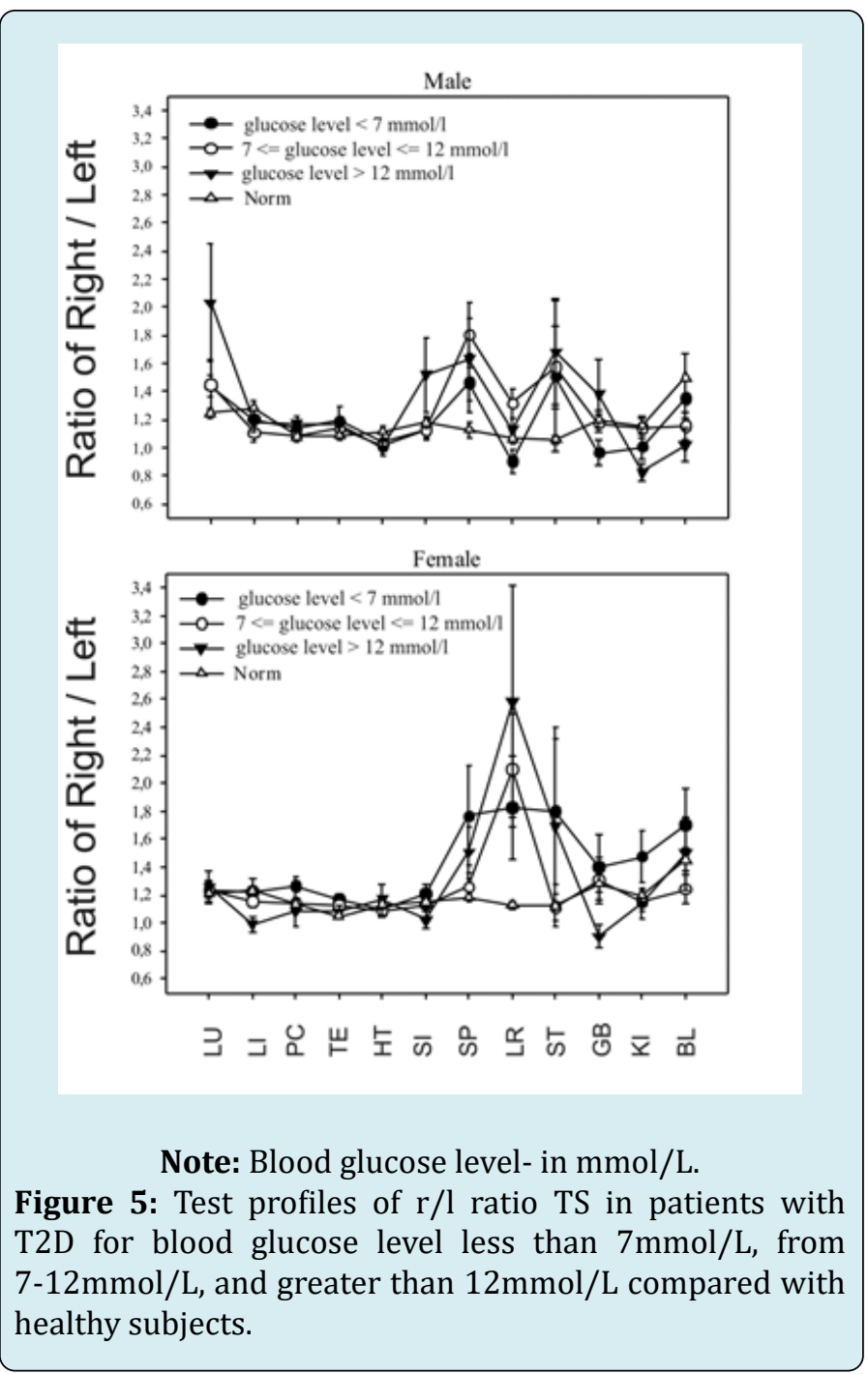

In general, a TS linear growth in the channels at LR, SP, and ST levels is observed in female patients. Linear significant dependencies of TS are identified in male patients in channels at LU, SP, LR, and KI. The difference in gender response are marked, especially in the channels of liver, stomach, and pancreas, which constitute the basic difference of T2D compared with healthy controls and type 1 diabetes $[8,10]$. The choice of the dose of the drug is especially relevant during long-term treatment, when addiction occurs and the previous dose no longer works adequately.

Let's consider how this scoring system works, for example, with T2D. Patient M ... B, 56 years old, has been suffering from type 2 diabetes for about 8 years. Overweight $-B M I=32.4$. The average sugar values in recent years are $8-12 \mathrm{mmol} / \mathrm{l}$. Treatment - diet therapy, MedFormin 500mg x 3 times a day. To reduce the level of glycemia in the last 2 weeks, I began to do additional injections of insulin of medium duration Novorapid from 2 to 8 units, depending on the level of glycemia.

Table 3 shows the regression dependences of the TS channels on the capillary blood sugar level based on 32 measurements at different times of the day before and 1-3 hours after insulin injection during the maximum activity of the drug.

\begin{tabular}{|c|c|c|c|c|}
\hline $\boldsymbol{A C}$ & $\boldsymbol{b}$ & $\boldsymbol{S E}$ & $\boldsymbol{T}$ & $\boldsymbol{p}$ \\
\hline CONSTANT & 32,43 & 6,12 & 4,26 & 0,001 \\
\hline LUr & $-6,17$ & 2,11 & $-3,10$ & 0,021 \\
\hline STr & $-6,58$ & 1,73 & $-3,79$ & 0,001 \\
\hline PCl & $-5,24$ & 2,12 & $-2,18$ & 0,043 \\
\hline SPr & 6,14 & 1,73 & 4,19 & 0,001 \\
\hline LRr & 5,27 & 2,21 & 2,14 & 0,028 \\
\hline STl & 8,72 & 3,87 & 3,12 & 0,017 \\
\hline KIr & $-5,11$ & 2,17 & $-2,28$ & 0,048 \\
\hline BLl & $-7,12$ & 2,51 & $-3,87$ & 0,001 \\
\hline
\end{tabular}

R-squared=79,2 \%. n-32

Table 3: Regression model of the effect of glycemia on the channels in the sample $\mathrm{p}<0.05$.

Table 4 shows the regression dependences of the TS channels on the insulin dose. Both models, which included only channels with significant dependencies $(\mathrm{p}<0.05)$, have high indicators of the polygon of explainable variance. In general, out of 8 channels associated with the level of glycemia, 7 have connections opposite in sign or along the side of the branches with the effect of insulin on the channels. Thus, the adhesion coefficient is $\mathrm{C}=8 / 7=1.14$, which reflects a fairly high interaction. The KIr channel remains unrelated 


\section{International Journal of Pharmacognosy \& Chinese Medicine}

to the effect of glycemia. According to the action of insulin, only the SIr channel is not associated with the influence of

glycemia, which reflects the small side effects of this drug.

\begin{tabular}{|c|c|c|c|c|}
\hline $\boldsymbol{A C}$ & $\boldsymbol{b}$ & $\boldsymbol{S E}$ & $\boldsymbol{T}$ & $\boldsymbol{p}$ \\
\hline CONSTANT & 13,58 & 1,69 & 8,03 & 0,001 \\
\hline LRr & $-2,21$ & 0,30 & $-7,19$ & 0,001 \\
\hline STr & 1,37 & 0,22 & 6,04 & 0,0011 \\
\hline BLr & 0,15 & 0,02 & 5,47 & 0,0002 \\
\hline LUr & 0,27 & 0,10 & 2,64 & 0,0226 \\
\hline SIr & 0,21 & 0,07 & 2,94 & 0,0132 \\
\hline HCr & $-0,16$ & 0,03 & $-4,17$ & 0,0016 \\
\hline SPr & $-1,01$ & 0,24 & $-4,10$ & 0,0017 \\
\hline
\end{tabular}

R-squared $=76,5 \%, \mathrm{n}-24$

Table 4: Regression model of the effect of insulin on channels with a sample of $p<0.05$.

\begin{tabular}{|c|c|c|c|c|c|c|c|c|c|c|c|c|c|}
\hline \multirow{2}{*}{ Time } & \multirow{2}{*}{$\mathrm{AC} r / \mathbf{l}$} & \multicolumn{12}{|c|}{$\mathrm{AC}$} \\
\hline & & LU & LI & PC & TE & HT & SI & SP & LR & ST & GB & KI & BL \\
\hline \multirow{2}{*}{ 13:30 } & Right & 7 & 7 & 4 & 6 & 4 & 4 & 21 & 18 & 5 & 20 & 16 & 37 \\
\hline & Left & 13 & 9 & 6 & 5 & 5 & 6 & 14 & 12 & 9 & 8 & 10 & 19 \\
\hline \multicolumn{2}{|c|}{$\mathrm{K}$ asymmetry $\mathrm{r} / \mathbf{l}$} & 0.53 & & 0.66 & & & & 1.5 & 1.5 & 0.55 & 2.5 & & 1.9 \\
\hline \multirow{2}{*}{$15: 30$} & Right & 11 & 6 & 6 & 5 & 5 & 8 & 9 & 7 & 7 & 10 & 12 & 14 \\
\hline & Left & 8 & 8 & 5 & 4 & 5 & 6 & 7 & 8 & 7 & 11 & 15 & 13 \\
\hline \multicolumn{2}{|c|}{$K$ asymmetry $\mathrm{r} / \mathrm{l}$} & 1.37 & & 1.2 & & & & 1.28 & 0.87 & 1 & 0.9 & & 1.1 \\
\hline
\end{tabular}

Table 5: Akabane test parameters before and after Novorapid injection-5 units.

In Table 5 shows the results of the Akabane test before the injection of Novorapid (13:30) and 2 hours after the meal, before which there was the injection. In the first case, the test was made at capillary blood sugar values of $16 \mathrm{mmol} / \mathrm{L}$, and the second at $9 \mathrm{mmol} / \mathrm{L}$. In the first case, there was a high coefficient of asymmetry $(\mathrm{K})$ in the LU, SP, LR, GB, ST, BL channels, which leveled out significantly under the targeted action of insulin. This, in turn, led to a significant decrease in the level of glycemia, because high asymmetry in the channels is the main manifestation of high glycemia [14]. Thus, this technique and the proposed evaluation criteria have shown their effectiveness.

\section{Discussion}

Usually, the assessment of drug action is based on the results of long and painstaking work, which usually starts with animals and ends with a group of test patients, which usually takes many years and requires a large investment. However, these measures do not guarantee that the drug will give the planned effect at the individual level.

Therefore, there is an urgent need to create an effective system for evaluating each drug at an individual level based on new physical principles, one of which this article is devoted to. For the first time, it is proposed to use the AC system as an information system, which exists in all living organisms and plants, and its information properties have not yet been properly evaluated by modern science [1923]. The proposed technique is simple to execute and can be performed by the patient himself using simple technical means www.chimaster.me. The principle of drug selection described in the article works not only at the insulin level in diabetes. It can be used to assess the effects of a wide variety of drugs in a variety of diseases. It is based on the fundamental laws of symmetry/asymmetry in nature, which also apply to humans.

In the future, with the development of this technology, it would be advisable to create bioenergy passports for various 


\section{International Journal of Pharmacognosy \& Chinese Medicine}

drugs. Thus, comparing their profile of activity at the level of acupuncture channels with the profile of a particular patient, it would be possible to select in advance the most appropriate individual medicine on the principle of "key to lock" based on the adhesion coefficient C.

As for the adequate dose and time of taking the medication, then of course, the current assessment system based on the Akabane test results is quite time consuming to execute. Recently, we have received good results in monitoring the activity of the AC based on the "pulse diagnosis" system. To do this, you can use a heart rate sensor, for example, in a watch or a special bracelet for continuous monitoring AC based on a special assessment of the phase and spectral parameters of the pulse wave. This information is processed in a Smartphone on a special program with the construction of graphs of the asymmetry of certain AC constantly in real time. Thus, it is very convenient to track the optimal dose and time of taking the drugs.

Thus, the development of this direction will allow creating many useful applications and high-tech devices not only for pharmacology, but also for medicine in general.

\section{Disclosure Statement}

The authors declare that they have no conflicts of interest and no financial interests related to the material of this manuscript.

\section{References}

1. Wagemans J (1997) Characteristics and models of human symmetry detection. Trends in Cognitive Sciences 1(9): 346-352.

2. Royer FL (1981) Detection of symmetry. J Exp Psychol Hum Percept Perform 7: 1186-1210.

3. Bouissou C, Petitjean M (2018) Asymmetric Exchanges. Journal of Interdisciplinary Methodologies and Issues in Science 4: 1-18.

4. Marola G (1989) On the detection of the axes of symmetry of symmetric and almost symmetric planar images. IEEE Trans Pattern Anal Mach Intell 11 (1): 104-108.

5. Muzhikov V, Vershinina E, Muzhikov R, Nikitin K (1956) Structure of Interchannel and Five Primary Elements Connections According to the Test of Akabane. Akabane K. Method Oi Hinaishin. Tokyo.

6. Muzhikov VG, Nozdrachev AD, Bogdanov NN (2005) Discrete Thermopuncture Test. Report 1 Reflexology 4(15): 23-28.
7. Muzhikov V, Vershinina E, Belenky V, Muzhikov R (2016) Significant heat sensitivity increase detected in various types of diabetes mellitus patients by Akabane test for use of management of diabetic patients. Acupunct Electrother Res 41(1): 1-19.

8. Valery Muzhikov, Elena Vershinina, Ruslan Muzhikov, Kirill Nikitin (2018) Thermopuncture for the diagnosis, monitoring, and treatment of patients with type 2 diabetes. Journal of Acupuncture and Meridian Studies 11(5): 323-331.

9. Muzhikov V, Vershinina E, Muzhikov R (2017) System of Thermopuncture Diagnostics and Monitoring of Patients with Type 1 Diabetes. J Altern Complement Integr Med 3: 2-7.

10. Valery Muzhikov, Elena Vershinina and Ruslan Muzhikov (2018) Opportunities of the Akabane Test for Diagnosis and Monitoring of Patients with Type 2 Diabetes. Journal of Diabetes and Metabolism 9: 2.

11. Muzhikov VG (2000) Theory and practice of the thermal acupuncture channel diagnostics and treatment St Petersburg Petrovsky Fund.

12. Muzhikov V (2003) Introduction to the energoskopia of man. ISBN 90-5179-103-8, Gopher Publishers 412.

13. Muzhikov VG, Lesogor VM, Reukov AS (2000) System of Monitoring and Correction in the Rehabilitation of Patients Suffering from Diabetes Mellitus. Methodical recommendations for doctors by the Medico-Social Expert Bureau of the Ministry of Labour and Social Development of the Russian Federation. CBNTI N 12.

14. Muzhikov VG Patent of Germany N 202010018 135.6. Device for reflex correction of functional infringements of the organism.

15. Muzhikov V, Vershinina E, Belenky V, Muzhikov R (2018) Assessing the links between anthropometrics data and Akabane test results. Journal of Acupuncture and Meridian Studies 11(1) 31-38.

16. Muzhikov V, Vershinina E, Muzhikov R, Nikitin K (2019) The Method Of Individual Assessment of The Action of Insulin and Its Adequate Dose in Diabetes Mellitus. World Journal of Pharmaceutical Research (WJPR) 8(7): 176-205.

17. MuzhikovV,VershininaE, Muzhikov R (2020)Possibilities of the Akabane Test and Cosinor Analysis to Predict the Level of Glycemia and the Individual Selection of Drugs and their Doses in the Treatment of Diabetes. Biomedical Journal of Scientific \& Technical Research (BJSTR) 29(3): 


\section{International Journal of Pharmacognosy \& Chinese Medicine}

502-509.

18. Umezawa H (1993) Advanced Field Theory: Micro, Macro, and Thermal Physics AIP, New York.

19. Blasone M, Jizba P, Vitiello G (2011) Quantum Field Theory and its Macroscopic Manifestations. Imperial College Press, London.

20. Del Giudice E, De Ninno A, Flesichmann M, Mengoli G, et al. (2006) Coherent quantum electrodynamics in matter. Electromagn Biol Med 25(3): 522-530.

21. Del Giudice E, Preparata G, Vitiello G (1988) Water as a free electric dipole laser. Phys Rev Lett 61(9): 10851088.

22. Brizhik L (2015) Influence of electromagnetic field on soliton mediated charge transport in biological systems. Electromagn Biol Med 34(2): 123-132. 